\title{
Visual form perception is a function of the visual cortex
}

\author{
DAVID LAVOND, MARY G. HATA. THACKERY S. GRAY, CHERI L. GECKLER, \\ PATRICIA M. MEYER, and DONALD R. MEYER \\ Laboratory of Comparative and Physiological Psychology, Ohio State University \\ Columbus, Ohio 43212
}

\begin{abstract}
Rats prepared with serial visual-cortical ablations can relearn the horizontal-vertical stripes discrimination problem if given interoperative training. However, they fail to discriminate between obliquely oriented stripes. The findings are discussed in relation to the concept that pattern perception is completely dependent upon the integrity of cortically related systems. Furthermore, the criteria for constructing pattern discriminanda are outlined, and reasons are presented for abandoning some of the classical stimuli used to test form perception in striate-extra-striate preparations.
\end{abstract}

It is thoroughly established that if rats are given training on a black-white discrimination problem and then subjected to bilateral ablations of the posterior cortex, they show no postoperative retention of performance of the problem but can nonetheless relearn it. Lashley (1935) believed that relearning of the problem was a function of subcortical systems, and his concept has since been supported by Horel, Bettinger, Royce, and Meyer (1966) and Meyer, Yutzey, Dalby, and Meyer (1968). The latter studies have shown that the problem is relearnable by rats with complete neocortical ablations, regardless of whether the ablations are performed with one-stage or two-stage procedures.

More recent research has been directed toward assessments of the variables which govern the rate at which the problem is relearned by rats with posterior ablations. One of the approaches has involved the use of procedures in which the animals are trained on the problem, subjected to unilateral posterior cortical ablations, then given interoperative retraining on the problem, subjected to second-stage ablations of the contralateral cortex, and then re-retrained on the problem. When this serial unilateral method is employed, the problem is finally relearned at a rate that is faster than the rate at which the problem is relearned by one-stage bilateral posterior preparations, or by subjects with two-stage posterior ablations which receive no interoperative practice (Glendenning, 1972; Kircher, Braun, Meyer, \& Meyer, 1970; Petrinovich \& Carew, 1969; Thompson, 1960; cf. Meyer \& Meyer, 1977, for review).

This research was supported, in part, by Grant MH-06211 from the National Institute of Mental Health to Donald R. Meyer and Patricia M. Meyer. The first author is a University Fellow and the second and third authors are Teaching Associates at the Ohio State University.
The question with which we are concerned in this study is whether the unilateral serial procedure, when combined with interoperative retraining, will bring about inductions of recoveries of performances of visual-pattern problems. Lashley believed that visualpattern learning was mediated by the visual cortex (Lashley, 1931; Lashley \& Frank, 1932), and that there was no recovery of the function, provided the subjects that were studied were prepared with lesions of the cortex that brought about complete degeneration of the dorsal lateral geniculate nuclei (LGNd) (Lashley, 1939). Although our own studies have supported his conclusions (Horel, Bettinger, Royce, \& Meyer, 1966; Jonason, Lauber, Robbins, Meyer, \& Meyer, 1970), they have recently been challenged by experiments of Spear and Barbas (1975), who showed that rats could relearn a horizontal-vertical stripes discrimination (HV) if the subjects were prepared with successive unilateral ablations of visual areas 17-18-18A and given interoperative training on the HV task. Also, Spear and Barbas (1975) observed that two rats with one-stage ablations of the same visual areas were able to relearn the problem, but only after very extensive retraining on the problem.

The latter observations led Spear and Barbas (1975) to argue that the findings of Horel et al. (1966) probably were negative because the subjects had not been given a sufficient amount of postoperative retraining on the problem. However, there were other differences between the methods of the two experiments. We now report the findings of two investigations in which we have systematically examined the results of the Spear and Barbas study. In Experiment 1, we compared the performances of subjects with radical posterior ablations, testing them either with the HV problem, as in the work of Spear and Barbas, or with the oblique-stripes discrimination problem (OB) used in the investigations of Horel 
et al. (1966) and Jonasen et al. (1970). In Experiment 2 , the subjects were prepared with restricted posterior ablations, and their final oblique-stripes performances were studied as a function of whether or not they were given interoperative training on that problem. In both investigations, two-stage procedures were employed in the production of the lesions, for we wished to maximize the probabilities that functional recoveries would be found. Also, the subjects of both experiments were given extensive postoperative retraining following their second-stage ablations so that there would be no question as to whether the animals had been retrained enough.

\section{EXPERIMENT 1}

\section{Method}

Subjects. The subjects were 20 adult Long-Evans male hooded rats, 90-120 days of age at the time the experiment began. The animals were housed individually and maintained on an ad-lib food and water schedule. They were randomly divided into two groups of 10 which were then designated as the $\mathrm{HV}$ and $\mathrm{OB}$ groups.

Apparatus and Procedures. The animals were trained in a Thompson-Bryant (1955) apparatus. It was basically the same as the apparatus used in the experiments of Horel et al. (1966), Jonason et al. (1970), and Spear and Barbas (1975). The apparatus consisted of a startbox, a choice compartment, and a goalbox. The first two compartments were separated by a small opaque guillotine door. The choice compartment and the goalbox were separated by a wall which contained two doorways $(9 \times 9 \mathrm{~cm}$ each, and $7.6 \mathrm{~cm}$ apart). The startbox and choice compartment were illuminated by a group of small overhead fluorescent lamps mounted on transparent roofs. The floors of the startbox and choice compartment consisted of a grid of stainless steel bars that was used to deliver motivating footshocks to the subjects.

The stimuli were patterns on the surfaces of the doors that were fitted into the two doorways. The discriminanda for the HV problem consisted of alternating black $(.1-\mathrm{mL})$ and white $(3.0-\mathrm{mL})$ stripes $(2.6 \mathrm{~cm} /$ cycle $)$ which were either vertical or horizontal. The discriminanda for the $\mathrm{OB}$ problem consisted of alternating black and white stripes $(2.5 \mathrm{~cm} /$ cycle $)$ at an orientation of $45^{\circ}$ or $135^{\circ}$

The HV problem was presented with a set of four doors. Each door consisted of either four black and three white stripes or three black and four white stripes. On any given trial, a door with a horizontal pattern was paired with one or the other of the doors with a vertical pattern, and hence four combinations of doors were employed. The pairings of the doors were governed by a Gellerman series, and the left-right positions of the horizontal and vertical patterns were governed by another such series.

The OB problem was presented with a single pair of doors. There were 10 stripes within each pattern, and the relative positions of the black and white stripes were altered on a trial-totrial basis by rotations of the doors through $180^{\circ}$. Thus, although only two doors were employed, the doors were paired in four different ways as a means of controlling for solutions of the problem in terms of local flux or local contour (cf. Ritchie, Meyer, \& Meyer, 1977). As in presentations of the HV problem, the variations in pairings of the doors and the left-right positions of two discriminanda were governed by a Gellerman series (1933).

Prior to pretraining, each of the rats was handled for at least 5 min per day for a total of 5 days. On Day 6, each rat was permitted to explore the apparatus for $15 \mathrm{~min}$ while all of the doors were removed. On Day 7, the guillotine door was replaced, and the animals were trained to avoid shock by running into the goalbox. On the next day, the pretraining doors, made of thin slats of black plastic, were placed in the doorways that led into the goalbox. The rat was then pretrained to exit from the startbox by knocking down a pretraining door. The incentive was avoidance of shocks to the feet (.2-.4 mA), set at the minimally effective level for each subject. The animals were allowed approximately $15 \mathrm{sec}$ after the startbox door was raised before they received a shock. After entering the runway, an interval of approximately $25 \mathrm{sec}$ intervened before shock administration.

On Day 8, preoperative HV or OB training was begun with the spaced-trial method of Glendenning (1972). Each of the subjects was run for 25 trials per day, with rests of 10 to $15 \mathrm{~min}$ between the 8 th and 9 th and the 16 th and 17 th trials. The incorrect stimulus door was locked at the rear, requiring the subject to exit from the choice compartment by knocking over the other stimulus door. An error was defined as a close approach to the negative stimulus door, and the subject was punished if it touched that door, stepped upon any of the first four bars in front of the negative stimulus, or hesitated immediately in front of the door. The subject was trained to a criterion of 9 correct responses in 10 trials, and criterion was typically achieved by the 3rd day of training on the problems.

Surgical and Retraining procedures. On Day 11, after each animal had learned the HV or OB problem, the first-stage surgical procedures were performed. At that time, each of the rats was subjected to a unilateral radical ablation of the posterior cortex. The lesions were intended to extend from the sagittal sinus to the rhinal sulcus, and from bregma back to the occipital pole. The lesions were produced by pia-stripping and by aspirations of the corners of the wound (cf. Meyer \& Meyer, 1971). They typically included areas $17,18,18 \mathrm{~A}, 7,20,29 \mathrm{c}$, and 40 , as defined by Krieg (1946). Hence, they were generally comparable to the lesions of the subjects of the Horel et al. (1966) and the Jonason et al. (1970) investigations, and destroyed all the areas which, according to Hughes (1977), receive a visually significant projection from nucleus lateralis posterior (NLP) thalami and/or LGNd. The surgery was performed with clean technique while the rat was anesthetized with sodium pentobarbital; after surgery, the animal was given a prophylactic broad-band antibiotic.

After 11 days' postoperative recovery, each rat was retrained $\left(R_{1}\right)$ for at least 50 trials on the visual-pattern problem that the subject had learned before the surgery. Additional retraining trials were given if the subject failed to re-reach the $9 / 10$ criterion within 50 trials, and the further retraining was continued until that criterion of performance was met.

Next, each subject was reoperated, and the still-intact contralateral posterior cortex was destroyed. The methods were the same as those followed during the first-stage procedures. The rat was once again permitted to recover from surgery for 11 days, and then, beginning on Day 35, it was once again retrained $\left(\mathbf{R}_{2}\right)$ on its problem. Two of the rats of the HV group and one of the rats of the $O B$ group did not receive the second-stage procedures. The eight surviving rats of the $\mathrm{OB}$ group were re-retrained on the OB problem for 850 trials ( 25 trials per day for 34 days), and the nine surviving rats of the HV group were reretrained on the $\mathrm{HV}$ problem for $\mathbf{4 2 5}$ trials. Thereafter, the rats of the $O B$ group were trained on a black-white discrimination problem with the same basic procedures, but with positive and negative stimulus doors which were white and black, respectively.

Histological procedures. When the neurobehavioral procedures were complete, the rats were overdosed with sodium pentobarbital and perfused intracardially with saline followed by $10 \%$ Formalin. The brains were then removed and stored in Formalin for at least 1 week. The extents of the cortical ablations were drawn on standard Lashley diagrams, and then the brains were frozen and sectioned at $30 \mu \mathrm{m}$. Every 10th section through the cortical lesion and every 5th section through LGNd were saved and stained with cresyl violet. Thereafter, conventional methods were followed for reconstruction of the lesions, with particular attention being paid to the question of whether any principal cells were still intact in the LGNd. 


\section{Results}

Behavioral results. Preoperatively, the HV subjects $(\mathrm{N}=10)$ learned the $\mathrm{HV}$ problem in $22.7 \pm 2.0$ / 25.0 (mean \pm standard error of the mean/median) trials to a criterion of 9 correct responses out of 10 trials. The $\mathrm{OB}$ subjects $(\mathrm{N}=10)$ learned the $\mathrm{OB}$ problem in $55.6 \pm 4.0 / 56.5$ trials to criterion. Thus, the rats of the HV group met their preoperative criterion of performance significantly faster than the subjects trained on the OB-discrimination problem.

After the subjects had recovered from their firststage ablations of the posterior cortex, the $\mathrm{HV}$ subjects $(\mathrm{N}=10)$ relearned the $\mathrm{HV}$ problem in 11.0 $\pm 2.7 / 7.5$ trials, and the $\mathrm{OB}$ subjects $(\mathrm{N}=10)$ relearned the $O B$ problem in $27.7 \pm 5.2 / 26.5$ trials. Hence, both groups took approximately half as many trials to relearn during $R_{1}$ as they did to learn their problem prior to surgery, and once again the subjects of the OB group required more training than the subjects of the HV group.

Figure 1 presents the results that were obtained when the subjects were retrained on their respective problems following their second-stage ablations. The HV subjects relearned their problem at a reasonably rapid rate, and during the last 100 trials of $R_{2}$, their average score was $83.3 \% \pm 3.4 \%$ correct responses (range: 69\%-96\%). However, the OB subjects failed to make substantial progress toward solution of their problem after twice as many trials of re-retraining, and during the last 100 trials of $R_{2}$, their average performance was $44.3 \% \pm 3.1 \%$ correct responses (range: $35 \%-64 \%$ ). The OB subjects, although they were unable to master the $\mathrm{OB}$ problem, were able to learn the black-white problem in $32.7 \pm 5.3 / 26.0$ trials.

Histological results. Figure 2 presents the reconstructions of the brains of all of the subjects. Each of the diagrams presents an estimate of the surface

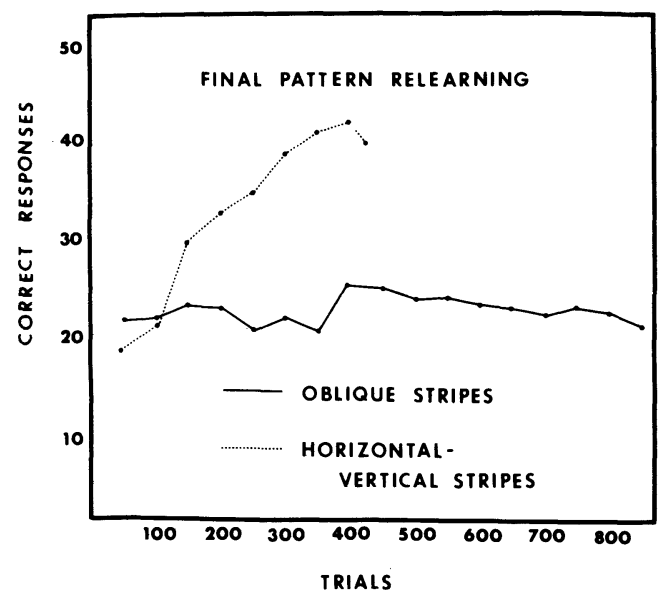

Figure 1. Correct responses in blocks of 50 trials $(2$ days) in final relearning of the pattern problems.

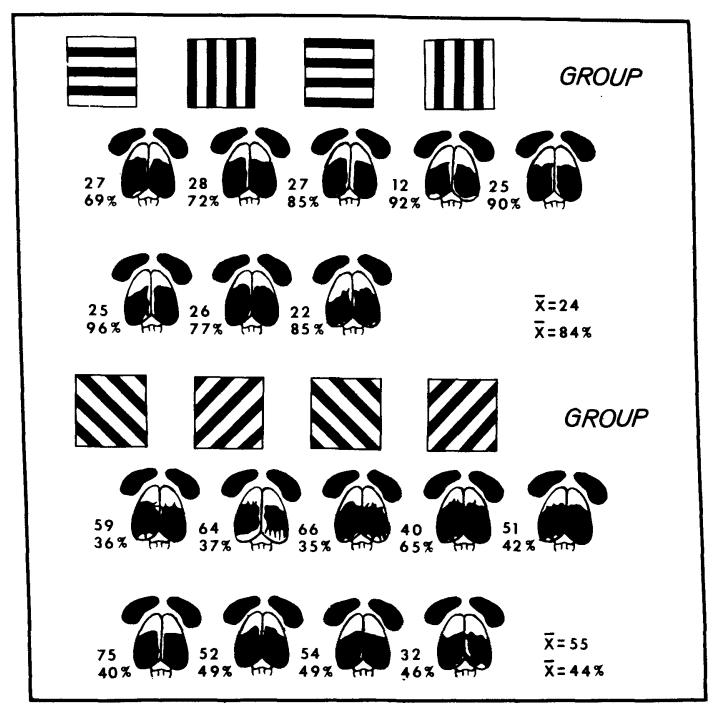

Figure 2. Surface diagrams of radical cortical ablations and representations of degeneration in the respective dorsal lateral geniculate bodies. The top number next to each figure is the number of trials taken to originally learn $(O L)$ the relevant pattern problem, and the bottom number is the percentage of correct trials made in the last 100 trials of performance in final learning.

extent of the cortical ablation and sections through the left and right LGNd in which the denerated regions are depicted in black. Adjacent to the diagrams are scores which give the number of trials each rat required for preoperative learning of its problem and the animal's percent correct responses during the final 100 re-retraining trials on its problem $\left(\mathbf{R}_{2}\right)$. The data indicate that the differences between the two groups' ultimate postoperative performances were not due to intergroup differences between the extent of the cortical ablations, and also that all of the subjects had sustained ablations which were large enough to bring about complete retrograde degeneration of the LGNd.

\section{EXPERIMENT 2}

In Experiment 2, we studied the performances of two further groups of animals. We attempted to restrict the lesions of the subjects to the striate and extra-striate cortex (17-18-18A). The question was whether subjects thus prepared can relearn the OB problem, and whether interoperative training on the problem facilitates relearning of the problem.

\section{Method}

Subjects. The subjects were 14 adult male Long-Evans rats which were housed and maintained as in the first experiment. There were two groups of 7 subjects each at the time that the study was begun.

Apparatus and Procedures. The apparatus and the basic procedures were the same as in Experiment 1. After handling and pretraining, all of the subjects were trained on the $\mathrm{OB}$ problem. 
Then they were prepared with first-stage unilateral lesions of 17-18-18A, and permitted to recover for 11 days. Thereafter, the subjects in what was designated as the IOT group were given interoperative retraining on the $\mathrm{OB}$ problem, and the remainder of the subjects (non-IOT) were permitted to rest for 3 more days. Then the second-stage ablations were performed, and after 11 days of recovery from surgery, the animals were given final retraining on the problem.

Final retraining on the problem continued until each subject reached a criterion of 18 correct responses out of 20 trials, or until it had failed to reach that criterion within 850 trials. The criterion of performance was thus the same criterion as that employed by Spear and Barbas (1975) in their study of recoveries of performance of the $\mathrm{HV}$ problem. Then, when the $\mathbf{R}_{\mathbf{2}}$ procedures were complete, all of the animals were trained on a black-white problem with the methods used for training the $O B$ subjects of Experiment 1.

Surgical and Histological procedures. The surgical and histological procedures were generally the same as the ones already described. However, the lesions were intended to be restricted to areas 17-18-18A, and hence to be very much smaller than the lesions of the subjects of the first experiment. First, area 17 was located by a stereotaxic measuring system devised by Hughes (1977). That area was destroyed, and then the ablation was expanded by $1.0 \mathrm{~mm}$ on its medial, lateral, and posterior aspects so that areas 18 and $18 \mathrm{~A}$ would also be destroyed. The anterior border was not extended because area 7 lies in that zone, and we wished to spare that area because we suspected that it might be important for recoveries of performances of visual-pattern problems by subjects with ablations of the striate and extrastriate cortex.

\section{Results}

Behavioral results. Preoperatively, the subjects of the IOT group learned the OB problem to a $9 / 10$ criterion in 47.3 $\pm 5.1 / 50.0$ mean \pm standard error of the mean/median trials. The subjects of the non-IOT group learned the $\mathrm{OB}$ problem to the same criterion in $38.9 \pm 6.0 / 40.0$ mean \pm standard error of the mean/median trials. The difference was not significant by a Mann-Whitney $U$ test. When pooled, the data for the two groups yielded a mean and median of $43.1 \pm 3.9 / 44.0$ trials for original learning of the problem.

The IOT subjects, when retrained on the problem following the first-stage surgical procedures, relearned the problem to a $9 / 10$ criterion in $38.4 \pm 3.3 / 35$ mean \pm standard error of the mean/median trials. Those scores were not significantly different from those obtained prior to operation and, in that respect, the finding was somewhat different from the finding of the first experiment.

Figure 3 presents the data for performance of the OB problem following completions of the secondstage surgical procedures. One of the subjects of the non-IOT group did not survive its second operation, and hence these results, which give the mean numbers of correct responses in successive blocks of 50 trials of training, are based upon performances of groups of six non-IOT and seven IOT subjects.

A two-factor mixed-design analysis of variance with repeated measures for one variable did not

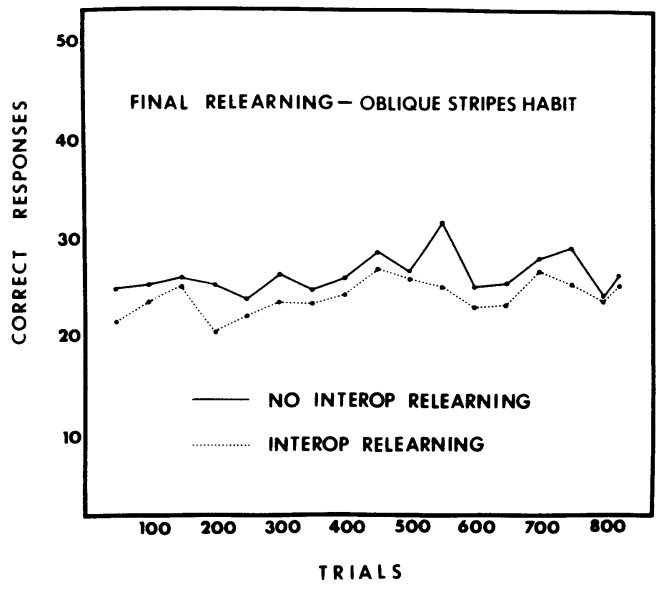

Figure 3. Mean number of correct responses in blocks of 50 trials ( 2 days) in final relearning of the oblique-stripes (OB) pattern problem. Group non-IOT, N $=6$, Group IOT, $\mathbf{N}=7$.

detect an intergroup difference $(\mathrm{F}=.83, \mathrm{df}=1,11$, $\mathrm{p}>$.05). A slight improvement was noted over days $(\mathrm{F}=2.00, \mathrm{df}=12,120, \mathrm{p}<.05)$, and a significant Group by Trials interaction was found in that improvement $(F=3.38$, df $=12,120, p<.001)$. The trials effect is explained by the fact that the rats, at the beginning of retraining, tended to hesitate in front of the doors and were punished for their hesitations. The interaction effect is explained by the fact that the subjects of the IOT group were somewhat slower than the other subjects, even though the difference could not be established by the tests performed on the preoperative learning scores.

The consequential findings were from the groups' behaviors during the last 100 trials of retraining on the problem. The mean for the subjects of the IOT group was approximately $52 \%$ correct responses (range: 33\%-74\%). The mean for the subjects of the other group was approximately $53 \%$ correct responses (range: $33 \%-76 \%$ ). Three of the 13 rats which survived their second operations eventually met the $18 / 20$ criterion of performance of the problem. The animals that met the criterion of relearning did so on Trials 260 and 826 (two rats, IOT group) and on Trial 826 (one rat, non-IOT group). However, the typical and long-term result was suppression of performance of the problem, and hence the findings of the second study were highly reminiscent of the OB results obtained in Experiment 1.

When the rats of both groups were subsequently given training on the black-white discrimination problem, they learned the problem to a $9 / 10$ criterion in $26.6 \pm 5.7 / 26.6$ mean \pm standard error of the mean/median trials. This result was also highly reminiscent of the findings of Experiment 1, in which we observed that the black-white problem can be learned very quickly by a rat that has been trained 
for many trials and days on a task beyond its competence. We think that it should also be of interest to students of black-white discrimination learning that the rates of initial acquisition of the problem were found in these experiments to be about the same for subjects with radical posterior ablations and subjects with much smaller injuries.

Histological results. Figure 4 shows, for each of the subjects, the extents of the cortical ablations and also the amounts of retrograde degeneration in LGNd. The numbers adjacent to each of the diagrams give the trials that each of the subjects required for preoperative learning of the problem (OL) and the animal's percent correct responses during the last 100 trials of retraining. Notably, in only one instance was it found that LGNd had undergone complete bilateral degeneration, and the exception was observed in a subject that was one of the better performers of the problem. We asked if there was any correlation between the extents of the ablations and the subjects' levels of performance on the last 100 trials, and we found that the rank-order correlation between extent of LGNd degeneration and performance, when corrected for ties, was -.21 . The rank-order correlation between performance and the scope of the cortical ablation, when corrected for ties, proved to be .00 .

\section{DISCUSSION}

Our findings have confirmed and extended the results of the experiments of Spear and Barbas (1975). Thus, we have shown that if rats are prepared with very large serial posterior ablations and are given interoperative training on the $\mathrm{HV}$ problem, they can relearn the HV problem following their second-stage ablations. Hence, we conclude that the Spear and Barbas outcomes were not a function of the fact that the lesions of their subjects were very much smaller than the lesions of the subjects of the Horel et al. (1966) and the Jonason et al. (1970) investigations.

However, we have also observed that rats prepared with two-stage radical posterior ablations are incapable of learning the oblique-stripes discrimination problem. Even with interoperative training, when their second-stage ablations are completed, they nonetheless exhibit complete and persistent impairments in performance of the problem. By that, we mean that performances are close to chance levels after 850 trials of training, or approximately 15 times the trials required for preoperative learning of the problem.

We asked if the outcomes could possibly have been a function of developments of stereotyped behaviors which might have kept the animals from learning other kinds of visual tasks (cf. Maier, 1943). Our method was to give the subjects final training on the

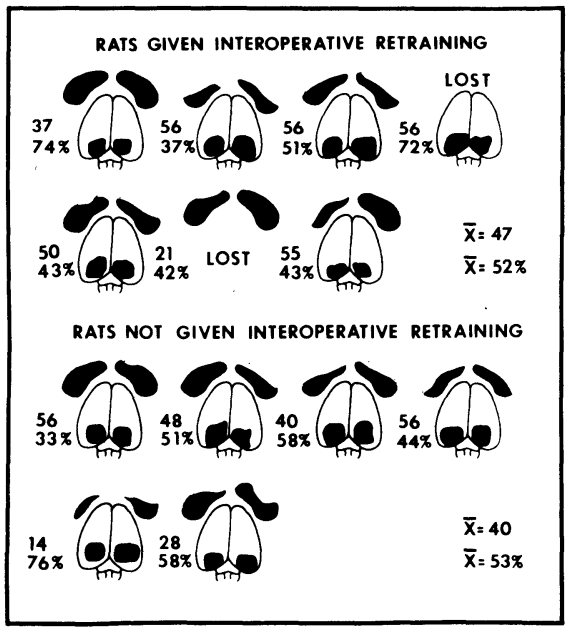

Figure 4. Surface diagrams of restricted cortical ablations of areas 17-18-18A and degeneration in the respective dorsal lateral geniculate bodies. The top number next to each figure is the number of trials taken to originally learn (OL) the relevant pattern problem, and the bottom number is the percentage of correct trials made in the last $\mathbf{1 0 0}$ trials of performance in final relearning of the oblique-stripes problem.

black-white discrimination problem, for we knew that the problem was learnable by rats with posterior lesions (cf. Meyer \& Meyer, 1977). We found that after having failed to learn the OB problem, our radical two-stage posterior preparations required only 33 spaced training trials to learn the black-white problem. Hence, we rejected the hypothesis that posterior subjects have residual capacities for learning the OB discrimination problem but fail to exhibit mastery of the problem because, in the early stages of retraining, they learn to react to presentations of the problem in ways that prevent them from detecting the discriminative cues.

We have also observed that if rats are prepared with very small serial ablations of the posterior cortex, the very great majority will also fail to relearn the OB discrimination problem. They will do so regardless of whether they are given interoperative training on the problem. We contrast those results with Spear and Barbas' findings (1975) with the HV discrimination problem. The differences could not have been a function of differences between the extents of the ablations, for Spear and Barbas found that the lesions of their subjects were sufficiently extensive to produce complete retrogade degenerations of LGNd in $70 \%$ of their cases. In our investigation, 10 of the 11 subjects for which we were able to present reconstructions of LGNd had evidence of spared principal cells in the dorsolateral crescents. However, despite the sparings we observed, our subjects' final mean performances were close to chance levels, and hence it was apparent that the outcomes of the studies were different because the discriminanda employed were different. 
We believe that the outcomes were what they were because a rat, when given training on the HV problem, is not necessarily forced to learn the problem by responding to differences between the slopes of the contours in the patterns. It is widely believed that alternative solutions are excluded by the fact that the HV patterns are equated with respect to overall contour and flux, and also that the problems are insoluble in terms of differences in local flux or contour provided that the black and white components of the patterns are varied on a trial-to-trial basis. However, although all of those requirements were met by Spear and Barbas and by us, we doubt that the methods used in either study accomplished their intended purposes.

If HV-pattern backgrounds are black, as they were in our experiments, a rat will find it hard to discriminate between the black apparatus background and a black stripe that lies along the edge of the pattern. Under such conditions, we believe that a form-blind rat could solve the problem as follows. First, we suppose that the rat would learn to scan the patterns in some consisunt manner, for example by inspecting the patterns in a left-to-right direction. If a left-right search is carried out by the rat and the $\mathrm{H}$ pattern is being scanned, the rat will first encounter an increment in flux that comes from the ends of the white stripes contained within the pattern. However, if the $\mathrm{V}$ cue is being scanned, the rat will first encounter an increment in flux that comes from a solid white stripe. Hence, inasmuch as a solid white stripe has a higher reflectance than an aggregate of the ends of black and white stripes of equal width, the rat can solve the problem by responding to local and momentary differences in flux. The foregoing argument is wholly independent of how the rat scans the HV patterns, for right-left, up-down, and downup strategies would also yield the flux differentials.

The OB problem is insoluble by such a strategy. Thus, all the edges of an OB pattern are alternately black and white, and the $O B$ problem is presented in a manner such that each particular segment of an edge is sometimes black and sometimes white. Hence, if the rat tries to use the strategy for solving the $O B$ problem, the strategy will fail because the increments or decrements of flux that it will see at transitions between the backgrounds and the edges of the patterns will be the same, stochastically, regardless of whether the animal is scanning a left-oblique or rightoblique pattern.

We think that the above considerations indicate that interoperative HV training facilitates recoveries of performance of that problem for reasons that will prove to be the same, when understood, as the reasons for the fact that interoperative training on the blackwhite problem will yield a protection of ultimate retention of the black-white discrimination habit (Barbas \& Spear, 1976; Glendenning, 1972; Meyer \&
Meyer, 1977; Thompson, 1960). Thus, although recoveries of HV performances by subjects given interoperative training on the HV problem are very much slower than recoveries of performances on the blackwhite discrimination problem, we believe that differences in rates do not imply that different mechanisms are involved. Instead, in our opinion, the differences are due to the fact that the momentary flux cues used by rats to solve the HV-discrimination problem are harder for decorticated animals to find than the cues derived from black and white doors. Perhaps the effect of a first-stage ablation is to increase the animal's reliance upon the momentary differential flux cues, but we think that those cues are also used by normal rats when they are trained on the $\mathrm{HV}$ problem.

Several studies (Dalby, Meyer, \& Meyer, 1970; Doty, 1971; Pavlov, 1927; Ritchie, Meyer, \& Meyer, 1976) have led us to formulate the following criteria in the design of pattern discriminanda: (1) the stimulus arrays must be equated for overall flux; (2) the arrays must be presented so that the problem is insoluble on the basis of local flux; (3) the arrays must be equated for total contour; and (4) the arrays must be presented so that they are insoluble in terms of local contour differences.

The OB-pattern stimuli meet the above criteria, and thus the discrimination can only be solved on the basis of the orientation of the stripes. We know from some recent experiments of Hughes (1977) that the problem can be learned by rats prepared with onestage bilateral ablations of the striate cortex. Also, Hughes has shown that bilateral ablations of nucleus lateralis posterior (NLP) have almost no effect upon performance of the habit if the striate cortex is intact, but nonetheless that lesions of NLP will markedly retard the rate of $O B$ learning by subjects also prepared with lesions of the striate cortex. Hence, he concluded that the NLP projections, although their visual significance is masked if the striate cortex is intact, convey information to the visual cortex which supplements the information it receives from the classical geniculate projections.

Hughes could not decide if the effect that he observed was due to the fact that the NLP ablations denervated areas 7 and/or 20 of Krieg (1946), or whether it resulted from the fact that the ablations also interrupted NLP projections to areas 18 and 18A. But the findings of the present experiments have shown that performance of the OB discrimination problem is suppressed by bilateral ablations of the cortex, even when the lesions are evidently confined to the striate and extrastriate cortex (areas 1718-18A). Hence, we believe that Hughes' results were due to destructions of the NLP projections to 18-18A, and particularly to $18 \mathrm{~A}$ because that region also receives a converging geniculate projection.

Our most general conclusion is that visual form 
perception (minimally defined as a capacity to see that contours have different orientations) is a function that depends, and absolutely so, upon the posterior neocortex. It is not, as Lashley (1939) thought, a function that is lost when the striate cortex is destroyed (Hughes, 1977), nor is it necessarily a function that can be recovered if a remnant of the lateral geniculate projections to the cortex is intact (present experiment). We think our definition of form perception is a useful one because, although we can imagine that visual form perception might be impaired in a subject that can still detect the orientations of edges, we find ourselves unable to imagine that the converse circumstance could possibly exist. The definition also is important because it is clear that certain kinds of spatial vision are intact after injuries to the posterior cortex, and that those capacities will sometimes permit a form-blind animal to learn discriminations which seem to be tests of form perception (cf. Ritchie, Meyer, \& Meyer, 1976).

Although we do not propose to comment at length upon the results of studies of the problem that have been carried out with other mammals, we nonetheless will say that we know of no convincing exceptions to the rules established for the rat. Thus, in our judgment, the putative exceptions have invariably been found in experiments in which the tests employed were not stringent tests of form perception. We suggest that those exceptions would quickly disappear if the outcomes of the studies were reevaluated through the use of the OB problem, for we think that the OB problem has much to recommend it as a test of capacities for orientational discriminations. While the problem is harder than the HV problem for a normal animal to learn, an average rat can learn it in about 2 days with training in the spaced-trial method. We have studied the problem in considerable detail and have found no trick solutions to it, which cannot be said for the very great majority of classical tests of visual form perception. Those tests worked well for Lashley (1939), but they only worked because his tests were for a very few trials; had he trained his subjects longer, he would undoubtedly have found that his rats could learn all of the problems in the set that he employed. They are still worth reviewing as examples of problems of the kinds which we think should be abandoned, and particularly so when, through the use of simple methods, the flaws in those tests can be avoided.

\section{REFERENCES}

Barbas, H., \& Spear, P. D. Effects of serial unilateral and serial bilateral visual cortex lesions on brightness discrimination relearning in rats. Journal of Comparative and Physiological Psychology, 1976, 90, 279-292.

Dalby, D. A., Meyer, D. R., \& Meyer, P. M. Effects of occipital neocortical lesions upon visual discriminations in the cat. Physiology \& Behavior, 1970, 5, 685-692.

Doty, R. W. Survival of pattern vision after removal of striate cor- tex in the adult cat. Journal of Comparative Neurology, 1971, 143, 341-370.

Gellerman, L. W. Chance orders of alternation stimuli in visual discrimination experiments. Journal of Genetic Psychology, 1933, 42, 207-208.

GLENDENNING, R. L. Effects of training between two unilateral lesions of visual cortex upon ultimate retention of black-white habits by rats. Journal of Comparative and Physiological Psychology, 1972, 80, 216-229.

Horel, J. A., Bettinger, L. A., Royce, G. J., \& Meyer, D. R. Role of neocortex in the learning and relearning of two visual habits by the rat. Journal of Comparative and Physiological Psychology, 1966, 61, 66-78.

Hughes, H. C. Anatomical and neurobehavioral investigations concerning the thalamo-cortical organization of the rat's visual system. Journal of Comparative Neurology, 1977, 175, 311-336.

Jonason, R. K., Lauber, S. M., Robbins, M. J., MeYer, P. M., \& MEYER, D. R. Effects of amphetamine upon relearning pattern and black-white discriminations following neocortical lesions in rats. Journal of Comparative and Physiological Psychology, 1970, 73, 47-55.

Kircher, K. A., Braun, J. J., Meyer, D. R., \& Meyer, P. M. Equivalence of simultaneous and successive neocortical ablations in production of impairments of retention of black-white habits in rats. Journal of Comparative and Physiological Psychology, 1970, 71, 420-425.

KRIEG, W. J. S. Connections of the cerebral cortex. I. The albino rat. A. Topography of the cortical areas. Journal of Comparative Neurology, 1946, 84, 221-275.

LASHLEY, K. S. The mechanism of vision: IV. The cerebral areas necessary for pattern vision in the rat. Journal of Comparative Neurology, 1931, 53, 419-472.

LASHLEY, K. S. The mechanism of vision: XII. Nervous structures concerned in habits based in reactions to light. Comparative Psychology Monographs, 1935, 11, 43-79.

LASHLEY, K. S. The mechanisms of vision: XVI. The functioning of small remnants of the visual cortex. Journal of Comparative Neurology, 1939, 70, 45-67.

LASHLEY, K. S., \& Frank, M. The mechanism of vision: VI. The lateral position of the area striata in the rat: A correction. Journal of Comparative Neurology, 1932, 55, 525-529.

MAIER, N. Two types of behavior abnormality in the rat. Bulletin of the Meninger Clinic, 1943, 7, 141-147.

Meyer, D. R., \& MeYer, P. M. Dynamics and bases of recoveries after injuries to the cerebral cortex. Physiological Psychology, 1977, 5, 133-165.

MeYer, P. M., \& MeYer, D. R. Neurosurgical procedures with special reference to aspiration lesions. In $\mathbf{R}$. Myers (Ed.), Methods in neuropsychology. New York: Academic Press, 1971.

Meyer, P. M., Yutzey, D. A., Dalby, D. A., \& MeYer, D. R. Effects of simultaneous septal-visual, septal-anterior and anterior-posterior lesions upon relearning a black-white discrimination. Brain Research, 1968, 8, 281-290.

Pavlov, I. P. Conditioned reflexes. London: Oxford University Press, 1927.

Petrinovich, L., \& Carew, T. J. Interaction of neocortical lesion size and interoperative experience in retention of a learned brightness discrimination. Journal of Comparative and Physiological Psychology, 1969, 68, 451-454.

Ritchie, G. D., Meyer, P. M., \& Meyer, D. R. Residual spatial vision of cats with lesions of the visual cortex. Experimental Neurology, 1976, 53, 227-253.

SPEAR, P. D., \& BARBAS, H. Recovery of pattern discrimination ability in rats receiving serial or one-stage visual cortex lesions. Brain Research, 1975, 94, 337-346.

Thompson, R. Retention of a brightness discrimination following neocortical damage in the rat. Journal of Comparative and Physiological Psychology, 1960, 53, 212-215.

Thompson, R., \& BRYANT, J. H. Memory as affected by activity of the relevant receptor. Psychological Report, 1955, 1, 393-400.

(Received for publication March 7, 1978; revision accepted September 1, 1978.) 